\title{
Representing free Boolean algebras
}

\author{
by \\ Alan Dow (North York, Ont.) \\ and Peter Nyikos (Columbia, S.C.)
}

\begin{abstract}
Partitioner algebras are defined in [2] and are natural tools for studying the properties of maximal almost disjoint families of subsets of $\omega$. In this paper we investigate which free algebras can be represented as partitioner algebras or as subalgebras of partitioner algebras. In so doing we answer a question raised in [2] by showing that the free algebra with $\aleph_{1}$ generators is represented. It was shown in [2] that it is consistent that the free Boolean algebra of size continuum is not a subalgebra of any partitioner algebra.
\end{abstract}

1. Introduction and main result. Two subsets of the integers, $A, B$, are almost disjoint if $A \cap B$ is finite. Similarly, $A$ and $B$ are almost equal, denoted by $A={ }^{*} B$, if $(A \backslash B) \cup(B \backslash A)$ is finite, and $A$ is almost contained in $B$ is denoted by $A \subset^{*} B$.

For any almost disjoint family $\mathcal{M}$ of subsets of $\omega$, a set $X \subset \omega$ is a partitioner of $\mathcal{M}$ if $(\forall M \in \mathcal{M})\left(X^{*} \supset M \vee X \cap M={ }^{*} \emptyset\right)$. The partitioner algebra, $\mathcal{B}_{\mathcal{M}}$, corresponding to $\mathcal{M}$ is the quotient of $\mathcal{P}_{\mathcal{M}}$ by the ideal, $\mathcal{I}_{\mathcal{M}}$, generated by $\mathcal{M}$, where $\mathcal{P}_{\mathcal{M}}$ is the set of partitioners of $\mathcal{M}$. From now on, algebra will always mean Boolean algebra. An algebra $\mathcal{B}$ is said to be representable if it is isomorphic to a partitioner algebra for some mad (maximal almost disjoint) family $\mathcal{M}$. We will say that a set $Y$ splits a set $M$ if $M \cap Y$ and $M \backslash Y$ are both infinite. Note that $Y \notin \mathcal{P}_{\mathcal{M}}$ if and only if $Y$ splits some member of $\mathcal{M}$. See [2] for more details.

Partitioner algebras are also very important to topologists. A topological space $\Psi$ is a $\Psi$-space if

- $\Psi$ is locally compact and Hausdorff,

- $\Psi$ has a countably infinite dense subset $D$ of isolated points,

- $\Psi \backslash D$ is an infinite discrete (closed) subspace,

- every infinite subset of $D$ contains a subsequence which converges to some point of $\Psi \backslash D$.

Research of the first author supported by NSERC of Canada. 
If $\mathcal{M}$ is a mad family on $\omega$, it is clear how to construct a $\Psi$-space $\Psi(\mathcal{M})$ from $\mathcal{M}$. Indeed, we take $D=\omega$ and $\mathcal{M}$ itself forms the set $\Psi \backslash D$. For each $M \in \mathcal{M},\{M\} \cup M$ is a clopen set in which $M$ (as a subset of $\omega$ ) converges to the point $M$. These spaces are introduced and shown to have the above properties in [6]. It follows that $\Psi(\mathcal{M})$ embeds naturally into the Stone space of the partitioner algebra, which turns out to be the maximal zerodimensional compactification of $\Psi(\mathcal{M})$ usually denoted by $\zeta[\Psi(\mathcal{M})]$. Recall that $\beta[\Psi(\mathcal{M})]$ is the maximal compactification of $\Psi(\mathcal{M})$. For a space $X, \beta X$ is equal to $\zeta X$ if and only if $\beta X$ is zero-dimensional. There are $\Psi$-spaces, $\Psi$, for which $\beta \Psi$ is not zero-dimensional [10].

We let $\mathcal{P}(S)$ denote the power set of $S$. Let us call an ideal $\mathcal{I} \subset \mathcal{P}(\omega)$ a $\kappa$-tower if $\mathcal{I}$ is generated by a set of cardinality $\kappa$ which is a maximal well-ordered chain when ordered by inclusion mod finite. (Here "maximal" means maximal in $\mathcal{P}(\omega) \backslash\left\{A \subset \omega: A=^{*} \omega\right\}$.) Recall that $\mathfrak{t}$ is the least cardinal such that there is a $\mathfrak{t}$-tower.

LEMma 1.1. If there is a $\kappa$-tower then there is an ideal $\mathcal{I}$ which is a $\kappa$-tower such that $\mathcal{P}(\omega) / \mathcal{I}$ contains a copy of $\mathcal{P}(\omega)$.

Proof. Let $\mathcal{J}$ be an arbitrary $\kappa$-tower. Let $f$ be any finite-to-one function from $\omega$ to itself with the property that $\left|f^{-1}(n)\right|=n$ for each $n \in \omega$. Let $\mathcal{I}$ be the ideal generated by $\left\{f^{-1}[J]: J \in \mathcal{J}\right\}$. If $\left\{J_{\alpha}: \alpha<\kappa\right\}$ is the well-ordered chain which generates $\mathcal{J}$, then $\left\{f^{-1}\left[J_{\alpha}\right]: \alpha<\kappa\right\}$ is again a well-ordered chain which generates $\mathcal{I}$. To see that $\left\{f^{-1}\left[J_{\alpha}\right]: \alpha<\kappa\right\}$ is maximal, assume that $f^{-1}\left[J_{\alpha}\right] \subset^{*} I$ for each $\alpha<\kappa$. Let $J=f[I]$ and note that $J_{\alpha} \subset^{*} J$ for each $\alpha<\kappa$; hence $\mathcal{I}$ is a $\kappa$-tower. Finally, we can embed $\mathcal{P}(\omega)$ into $\mathcal{P}(\omega) / \mathcal{I}$ as follows. For each $n \in \omega$ we may view $f^{-1}(n)$ as a copy of the set $n=\{0,1, \ldots, n-1\}$. For each $A \subset \omega$, let $X_{A}$ be the subset of $\omega$ with the property that $X_{A} \cap f^{-1}(n)$ is exactly $A \cap n$ under the above identification.

COROLlary 1.2. There is a $\mathfrak{t}$-tower $\mathcal{I}$ such that $\mathcal{P}(\omega) / \mathcal{I}$ contains a copy of the free algebra on $\mathfrak{c}$ generators.

Proof. Clearly it follows from $\mathfrak{t}=\kappa$ that there are $\kappa$-towers. So apply the above lemma together with the fact that $\mathcal{P}(\omega)$ contains a copy of the free algebra with $\mathfrak{c}$ generators.

We shall need the following fundamental result from [2] (the result for countable algebras, $\mathcal{B}$, is due to Teresawa [10]). Recall that $\mathfrak{p}$ is the least cardinal such that there is a filter base of infinite subsets of $\omega$ for which there is no infinite set which is almost included in each member of the filter.

Proposition 1.3. If $\mathcal{B}$ is a subalgebra of $\mathcal{P}(\omega)$ consisting only of infinite sets and $|\mathcal{B}|<\mathfrak{p}$, then there is a mad family $\mathcal{M}$ such that $\mathcal{B} / \mathcal{I}_{\mathcal{M}}$ is the partitioner algebra for $\mathcal{M}$ (i.e. not only is $\mathcal{B}$ isomorphic to the partitioner 
algebra but it is essentially equal to it). Furthermore, it may be assumed that, for each filter $\mathcal{F} \subset \mathcal{B}$,

$$
\left|\left\{M \in \mathcal{M}:(\forall F \in \mathcal{F})\left(M \subset^{*} F\right)\right\}\right|=\mathfrak{c},
$$

Proof. Each ultrafilter on $\mathcal{B}$ is a filter of fewer than $\mathfrak{p}$ infinite subsets of $\omega$ and there are therefore many infinite subsets of $\omega$ which are almost contained in every member of this filter. Therefore a mad family of infinite subsets of $\omega$ can be found such that $\mathcal{B}$ is a subset of the partitioner algebra. Notice also that if $\mathcal{M}$ is any mad family with $\mathcal{B} \subset \mathcal{B}_{\mathcal{M}}$ then for each $M \in \mathcal{M}$, $\left\{B \in \mathcal{B}_{\mathcal{M}}: M \subset^{*} B\right\}$ generates an ultrafilter, $\mathcal{U}_{M}$, on $\mathcal{B}_{\mathcal{M}}$. One then uses a technique originally used by Mrówka [7] to modify the family so as to keep all of $\mathcal{B}$ as partitioners but to destroy the unwanted partitioners. The technique is to collapse certain pairs of the first family into single sets by simply taking the union of the pair. The resulting family will still be mad, and if for each pair that is collapsed the same ultrafilter on $\mathcal{B}$ is generated by each set in the pair, then every member of $\mathcal{B}$ will remain a partitioner. However, partitioners can be destroyed by choosing a pair which is split by that partitioner. It is clear that all unwanted partitioners can be killed in this way if $\mathcal{M}$ can be chosen so that for each partitioner $Y \in \mathcal{P}_{\mathcal{M}}$ and each ultrafilter $\mathcal{U}$ on $\mathcal{B},\left\{M \in \mathcal{M}: \mathcal{U}=\mathcal{U}_{M}\right.$ and $\left.|Y \cap M|=\aleph_{0}\right\}$ is either finite or has cardinality $\mathfrak{c}$.

This can be accomplished as follows. First of all we use the fact that Mrówka constructed a mad family with the property that every partitioner which meets infinitely many, actually meets $\mathfrak{c}$-many. Next, for each ultrafilter $\mathcal{U}$ of $\mathcal{B}$ choose an arbitrary infinite set $A_{\mathcal{U}}$ which is contained mod finite in every member of $\mathcal{U}$. Fix a "Mrówka" family, $\mathcal{M}_{\mathcal{U}}$, of subsets of $A_{\mathcal{U}}$. Then, by using the collapsing technique, we may choose a family $\mathcal{N}_{\mathcal{U}}$ of infinite sets such that $M \cap A_{\mathcal{U}} \in \mathcal{M}_{\mathcal{U}}$ for each $M \in \mathcal{N}_{\mathcal{U}}$ and which is maximal with respect to being almost disjoint among the set of all infinite sets which are contained mod finite in every member of $\mathcal{U}$. Since $\mathcal{B}$ has cardinality less than $\mathfrak{p}$ it follows that $\mathcal{M}=\bigcup\left\{\mathcal{M}_{\mathcal{U}}: \mathcal{U}\right.$ is an ultrafilter on $\left.\mathcal{B}\right\}$ is a mad family. We check that $\mathcal{M}$ has the desired property. If a partitioner $Y$ meets infinitely many members of $\mathcal{N}_{\mathcal{U}}$ then (since $Y$ is a partitioner) it must meet infinitely many members of $\mathcal{M}_{\mathcal{U}}$. But since this was a Mrówka family on $\mathcal{P}\left(A_{\mathcal{U}}\right)$, it follows that $Y$ meets $\mathfrak{c}$-many members of $\mathcal{M}_{\mathcal{U}}$.

It is not yet known whether the set-theoretic hypothesis " $\mathfrak{p}=\mathfrak{t}$ ", used below, is a theorem of ZFC ([11], [12]).

Theorem 1.4. If $\mathfrak{p}=\mathfrak{t}$, then for each $\kappa \leq \mathfrak{p}$, the free algebra with $\kappa$ generators can be represented.

Proof. Clearly the case $\kappa<\mathfrak{p}$ follows from Proposition 1.3 so let $\kappa=\mathfrak{p}=\mathfrak{t}$. Let $\mathcal{I}$ be any $\kappa$-tower such that $\mathcal{P}(\omega) / \mathcal{I}$ contains the free algebra 
with $\kappa$ generators. Let $\left\{X_{\alpha}: \alpha<\kappa\right\} \subset \mathcal{P}(\omega)$ be representatives for the generators for the above copy of the free algebra. Let $\left\{I_{\alpha}: \alpha<\kappa\right\}$ be a well-ordered (mod finite) family of generators for $\mathcal{I}$ with $I_{0}=\emptyset$. Since we may choose a subsequence and then re-index, we may assume that, for each $\alpha<\kappa$, the family $\left\{X_{\beta} \cap\left(I_{\alpha+1} \backslash I_{\alpha}\right): \beta \leq \alpha\right\}$ forms an independent family. Let us now replace each $X_{\alpha}$ by $X_{\alpha} \backslash I_{\alpha}$. The above properties of the family $\left\{X_{\alpha}: \alpha<\kappa\right\}$ are still valid for this revised family. For each $\alpha<\kappa$, let $\mathcal{X}_{\alpha}$ denote the subalgebra of $\mathcal{P}(\omega)$ which is generated by $\left\{X_{\beta}: \beta<\alpha\right\}$ and let $\mathcal{X}$ denote the algebra which is generated by $\left\{X_{\alpha}: \alpha<\kappa\right\}$.

Use Proposition 1.3, to produce a mad family $\mathcal{M}_{\alpha+1}$ on $\mathcal{P}\left(I_{\alpha+1} \backslash I_{\alpha}\right)$ for each $\alpha$ so that $\mathcal{M}_{\alpha}$ represents the algebra generated by $\left\{X_{\beta} \cap\left(I_{\alpha+1} \backslash\right.\right.$ $\left.\left.I_{\alpha+1}\right): \beta \leq \alpha\right\}$. Using the same principle as in Proposition 1.3, we may also choose an almost disjoint family, $\mathcal{M}_{\alpha}$, of subsets of $I_{\alpha}$, for limit $\alpha$, which we may think of as representing the algebra $\left\{X_{\beta}: \beta<\alpha\right\}$ restricted to $I_{\alpha} \backslash\left[\bigcup_{\beta<\alpha} I_{\beta}\right]$. More specifically, we may choose $\mathcal{M}_{\alpha}$ so that:

1) $M \cap I_{\gamma}={ }^{*} \emptyset$ for each $M \in \mathcal{M}_{\alpha}$ and $\gamma<\alpha$,

2) each $X_{\beta}, \beta<\alpha$, is a non-trivial partitioner of $\mathcal{M}_{\alpha}$,

3) if $Y$ is an infinite subset of $I_{\alpha}$ which is almost disjoint from each $I_{\gamma}$ for $\gamma<\alpha$, then there is an $M \in \mathcal{M}_{\alpha}$ which meets $Y$ in an infinite set, and

4) if $Y$ is any partitioner of $\mathcal{M}_{\alpha}$ then there is a $B \in \mathcal{X}_{\alpha}$ and a $\gamma<\alpha$ such that $B \cap\left(I_{\alpha} \backslash I_{\gamma}\right)$ and $Y \cap\left(I_{\alpha} \backslash I_{\gamma}\right)$ are equivalent modulo $\mathcal{I}_{\mathcal{M}_{\alpha}}$.

The only new idea required here is to accomplish 4$)$. First choose a family $\mathcal{M}$ of subsets of $I_{\alpha}$ satisfying 1)-3). That is, for each $M \in \mathcal{M}, M \cap I_{\beta}$ is finite for each $\beta<\alpha$, and $\mathcal{M}$ is maximal with respect to this property. In addition, as discussed following Proposition 1.3, we may ensure that for each ultrafilter $\mathcal{U}$ on the algebra $\left\{B \cap I_{\alpha}: B \in \mathcal{X}_{\alpha}\right\}$ and each partitioner $Y \subset I_{\alpha}$, the set $\left\{M \in \mathcal{U}_{\mathcal{M}}:|Y \cap M|=\aleph_{0}\right\}$ is either finite or has cardinality $\mathfrak{c}$. We define $\mathcal{M}_{\alpha}$ using an induction of length $\mathfrak{c}$ in which we pick pairs from $\mathcal{M}$ which we will "collapse" to form $\mathcal{M}_{\alpha}$ so as to ensure all partitioners satisfy condition 4). Indeed, let $Y \subset I_{\alpha}$ be a partitioner and suppose that for each $\beta<\alpha, Y \backslash I_{\beta}$ is not in the algebra generated by $\left\{X_{\gamma} \cap\left(I_{\alpha} \backslash I_{\beta}\right): \gamma<\alpha\right\} \cup \mathcal{M} \cup[\omega]^{<\omega}$. Let this algebra be denoted by $\mathcal{C}_{\beta}$ and let $\mathcal{C}=\bigcup_{\beta<\alpha} \mathcal{C}_{\beta}$. Let

$$
\begin{aligned}
& \mathcal{J}=\left\{X \cap I_{\alpha}: X \in \mathcal{X}_{\alpha} \text { and }(\exists \gamma<\alpha)\left(\left(X \cap I_{\alpha}\right) \backslash I_{\gamma} \subset^{*} Y\right)\right\}, \\
& \left.\mathcal{K}=\left\{X \cap I_{\alpha}: X \in \mathcal{X}_{\alpha} \text { and }(\exists \gamma<\alpha)\left(\left(X \cap I_{\alpha}\right) \backslash I_{\gamma}\right) \cap Y={ }^{*} \emptyset\right)\right\} .
\end{aligned}
$$

By our hypothesis on $Y, \mathcal{M} \cup \mathcal{J} \cup \mathcal{K} \cup\left\{I_{\gamma}: \gamma<\alpha\right\}$ generates an ideal such that we may choose an ultrafilter $\mathcal{U}$ on $\mathcal{C}$ which is disjoint from this ideal. Suppose that there is some $U \in \mathcal{U}$ such that $Y \cap U$ is almost disjoint from all but finitely many members, say $\mathcal{M}_{Y}^{0}$, of $\mathcal{M}_{\mathcal{U}}=\left\{M \in \mathcal{M}: M \subset^{*}\right.$ $U$ for each $U \in \mathcal{U}\}$. Then $\bar{U}=U \backslash \bigcup \mathcal{M}_{Y}^{0} \in \mathcal{U}$ and $\bar{U} \cap Y$ finite. However, this contradicts that $\bar{U} \notin \mathcal{K}$. Therefore $Y$, and similarly, $I_{\alpha} \backslash Y$, meets $\mathfrak{c}$ - 
many members of $\mathcal{M}_{\mathcal{U}}$. If some member of $\mathcal{M}$ is split by $Y$, then there is no need to do anything. If not, we choose a pair of elements, say $M_{0, Y}, M_{1, Y}$, of $\mathcal{M}$ such that:

- neither has been chosen at previous steps in the induction,

- $M_{0, Y} \subset^{*} U \cap Y$ for each $U \in \mathcal{U}$, and

- $M_{1, Y} \subset^{*} U \backslash Y$ for each $U \in \mathcal{U}$.

It follows that when we define $\mathcal{M}_{\alpha}$ to include the set $M_{0, Y} \cup M_{1, Y}$, the set $Y$ will not be a partitioner.

Now we have $\mathcal{M}_{\alpha}$, for $\alpha<\kappa$, with the following properties:

1) $M \in \mathcal{M}_{\alpha}$ implies that $M \subset^{*} I_{\alpha} \backslash I_{\beta}$ for each $\beta<\alpha$,

2) each $X_{\beta}$ is a partitioner of the mad family $\bigcup_{\alpha<\kappa} \mathcal{M}_{\alpha}$, and

3) for each partitioner $Y$ of $\bigcup_{\alpha<\kappa} \mathcal{M}_{\alpha}$, and for each $\alpha$, there is an $X$ in $\mathcal{X}_{\alpha}$ and a $\beta<\alpha$ such that $Y \cap\left(I_{\alpha} \backslash I_{\beta}\right)$ and $X \cap\left(I_{\alpha} \backslash I_{\beta}\right)$ are equivalent modulo $\mathcal{M}_{\alpha}$.

Now, of course, we would like to replace the third condition by the fact that $Y$ and $X$ are equivalent. This is not the case for sets of the form $\left(X \cap I_{\alpha}\right) \cup\left(X^{\prime} \backslash I_{\alpha}\right)$, where $X$ and $X^{\prime}$ are distinct members of $\mathcal{X}_{\alpha}$ and $\mathcal{X}$, respectively. To fix this we will now collapse some members of our mad family.

FACT 1.4.1. There are disjoint $\mathcal{M}_{\alpha, 0}$ and $\mathcal{M}_{\alpha, 1}$, for each $\alpha<\kappa$, so that $\mathcal{M}_{\alpha}=\mathcal{M}_{\alpha, 0} \cup \mathcal{M}_{\alpha, 1}$ and a function $f_{\alpha}$ from $\mathcal{M}_{\alpha, 0}$ into $\mathcal{M}_{\alpha+1,1}$ so that

$$
\left(\forall M \in \mathcal{M}_{\alpha, 0}\right)(\forall X \in \mathcal{X})\left(M \subset^{*} X \text { iff } f_{\alpha}(M) \subset^{*} X\right) .
$$

Proof of Fact 1.4.1. Recall that we have chosen the families $\mathcal{M}_{\alpha}$ so that, for each filter $\mathcal{F} \subset \mathcal{X}_{\alpha}$,

$$
\left|\left\{M \in \mathcal{M}_{\alpha}:(\forall F \in \mathcal{F})\left(M \subset^{*} F\right)\right\}\right|=\mathfrak{c} .
$$

For each such $\mathcal{F}$, this set may be partitioned into two pieces. Therefore, for each $\alpha<\kappa$, we may choose $\mathcal{M}_{\alpha, 0}, \mathcal{M}_{\alpha, 1} \subset \mathcal{M}_{\alpha}$ so that, for each $i \in\{0,1\}$,

$$
\left|\left\{M \in \mathcal{M}_{\alpha, i}:(\forall F \in \mathcal{F})\left(M \subset^{*} F\right)\right\}\right|=\mathfrak{c} .
$$

Since $X_{\beta} \cap I_{\alpha}={ }^{*} \emptyset$ for $\alpha \leq \beta$, it follows that the previous condition holds for each filter $\mathcal{F} \subset \mathcal{X}$.

For each $M \in \mathcal{M}_{\alpha, 0}$, let $\mathcal{F}_{M}$ be the filter of all $F \in \mathcal{X}_{\alpha}$ such that $M \subset^{*} F$. Now by simply well-ordering $\mathcal{M}_{\alpha, 0}$ we may inductively choose $f_{\alpha}(M) \in \mathcal{M}_{\alpha+1,1}$, for $M \in \mathcal{M}_{\alpha, 0}$, so that $f_{\alpha}(M) \subset^{*} F$ for all $F \in \mathcal{F}_{M}$. Similarly, we may ensure that the range of $f_{\alpha}$ is exactly $\left\{M \in \mathcal{M}_{\alpha+1,1}\right.$ : $M \cap X_{\alpha}$ is finite $\}$. Let $\mathcal{M}_{\alpha+1,2}=\left\{M \in \mathcal{M}_{\alpha+1,1}: M \subset^{*} X_{\alpha}\right\}$ and for $\alpha$ a limit let $\mathcal{M}_{\alpha, 2}=\mathcal{M}_{\alpha, 1}=\emptyset$. To complete the proof of Fact 1.4.1, we must define $\mathcal{M}_{\alpha, 0}$ and $\mathcal{M}_{\alpha, 1}$ for $\alpha=0$ and for $\alpha$ a limit. In all these cases, we just let $\mathcal{M}_{\alpha, 0}=\mathcal{M}_{\alpha}$ and $\mathcal{M}_{\alpha, 1}=\emptyset$. 
We define

$$
\mathcal{M}=\bigcup_{\alpha<\kappa}\left[\mathcal{M}_{\alpha, 2} \cup\left\{M \cup f_{\alpha}(M): M \in \mathcal{M}_{\alpha, 0}\right\}\right] .
$$

It remains to check that $\mathcal{B}_{\mathcal{M}}$ is isomorphic to $\mathcal{X}$. The first condition of Fact 1.4.1 guarantees that each $X \in \mathcal{X}$ is a partitioner of $\mathcal{M}$. For sets $A, B \subset \omega$, let $A=B(\bmod \mathcal{M})$ abbreviate the statement that there is a finite set $\mathcal{N} \subset \mathcal{M}$ such that, for $M \in \mathcal{M} \backslash \mathcal{N}, A \cap M$ is infinite iff $B \cap M$ is infinite.

FACT 1.4.2. For each $Y \in \mathcal{P}_{\mathcal{M}}$ and for each $\alpha<\kappa$ there is an $X \in \mathcal{X}$ such

$$
X \cap I_{\alpha}=Y \cap I_{\alpha}(\bmod \mathcal{M}) .
$$

Proof of Fact 1.4.2. By property 4) of the family $\mathcal{M}_{\alpha}$, there is an $X \in \mathcal{X}$ and a $\xi<\alpha$ such that $Y \cap\left(I_{\alpha} \backslash I_{\xi}\right)=X \cap\left(I_{\alpha} \backslash I_{\xi}\right)(\bmod \mathcal{M})$. Let $\xi$ be minimal such that $Y \cap\left(I_{\alpha} \backslash I_{\xi}\right)=X \cap\left(I_{\alpha} \backslash I_{\xi}\right)(\bmod \mathcal{M})$. Clearly we may assume that $\xi>0$ since $I_{0}=\emptyset$. Now choose $X^{\prime} \in \mathcal{X}$ so that there is a $\gamma<\xi$ so that $Y \cap\left(I_{\xi} \backslash I_{\gamma}\right)=X^{\prime} \cap\left(I_{\xi} \backslash I_{\gamma}\right)(\bmod \mathcal{M})$. By the minimality of $\xi, X \cap\left(I_{\xi} \backslash I_{\gamma}\right) \neq X^{\prime} \cap\left(I_{\xi} \backslash I_{\gamma}\right)(\bmod \mathcal{M})$ for all $\gamma<\xi$. Now at least one of $\left(X \backslash X^{\prime}\right) \cap\left(I_{\xi} \backslash I_{\gamma}\right)$ or $\left(X^{\prime} \backslash X\right) \cap\left(I_{\xi} \backslash I_{\gamma}\right)$ is not equivalent to zero modulo $\mathcal{M}$ for all $\gamma<\xi$. Assume it is $X \backslash X^{\prime}$ which is not; the other case is handled similarly. Therefore there is an $M \in \mathcal{M}_{\xi}$ such that $M \subset^{*} X \backslash X^{\prime}$, hence $f_{\xi}(M) \subset^{*} X \backslash X^{\prime}$. Since there are c-many such $M$, we may assume that $M \cup f_{\xi}(M)$ is not a member of the finite set which witnesses the hypothesized equivalences of $X$ or $X^{\prime}$ to $Y$. But now, since $f_{\xi}(M) \subset^{*} X, f_{\xi}(M) \subset^{*} Y$, whereas $M \subset^{*} X \backslash X^{\prime}$ implies that $M \cap Y$ is finite. It follows that $Y$ splits $M \cup f_{\xi}(M)$, contradicting that $Y \in \mathcal{P}_{\mathcal{M}}$.

Now we show that if $Y \in \mathcal{P}_{\mathcal{M}}$, then there is an $X \in \mathcal{X}$ which is equivalent to $Y$ modulo $\mathcal{M}$. By Fact 1.4.2 it is clear that we may choose, for each $\alpha<\kappa$, an $A_{\alpha} \in \mathcal{X}$ so that $Y \cap I_{\alpha}=A_{\alpha} \cap I_{\alpha}(\bmod \mathcal{M})$. We may suppose that $A_{\alpha} \in \mathcal{X}_{\alpha}$, since for all $X \in \mathcal{X}$ there is an $X^{\prime} \in \mathcal{X}_{\alpha}$ such that $X \cap I_{\alpha}=$ $X^{\prime} \cap I_{\alpha} \bmod \mathcal{M}$. Now by the pressing-down lemma, there is an $A \in \mathcal{X}$ so that $A_{\alpha}=A$ for $\kappa$-many $\alpha$ 's. Clearly $Y$ is equivalent to $A$ modulo $\mathcal{M}$.

COROLlary 1.5. The free algebra on $\aleph_{1}$ generators is representable.

Proof. If $\mathfrak{p}>\aleph_{1}$, then this follows from Proposition 1.3. If $\mathfrak{p}=\aleph_{1}$, then by $[9], \mathfrak{t}=\aleph_{1}$ and we apply the previous theorem.

It is shown in [4] that it is consistent to have a non-representable algebra of cardinality $\aleph_{1}$.

2. Subrepresentable algebras. As mentioned in the abstract, it is proven in [2] that it is consistent that the free algebra with $\mathfrak{c}$ generators is not even a subalgebra of any representable algebra. Let us now consider the 
problem of determining those $\kappa$ for which the free algebra with $\kappa$ generators is a subalgebra of a representable algebra.

It turns out that this property has a much simpler formulation. We will look at some topological equivalences in the next section. A family $\mathcal{A} \subset \mathcal{P}(\omega)$ is said to be splitting if for each infinite $Y \subset \omega$, there is an $A \in \mathcal{A}$ with $|Y \cap A|=|Y \backslash A|=\aleph_{0}$. Let us call a family $\mathcal{A}$ nowhere splitting if for each infinite $X \subset \omega$, there is an infinite $Y \subset X$ such that $Y$ is not split by $\mathcal{A}$.

Proposition 2.1. Let $\kappa$ be an infinite cardinal. The following are equivalent.

1. The free algebra on $\kappa$ generators is a subalgebra of some representable algebra.

2. There is an independent family $\mathcal{X}$ of cardinality $\kappa$ which is nowhere splitting.

3. There is a mad family $\mathcal{M}$ and an independent family $\mathcal{X}$ of cardinality $\kappa$ such that no member of $\mathcal{M}$ is split by any member of $\mathcal{X}$.

Proof. It is very easy to check that each of the first two statements is equivalent to the third.

Recall that $\mathfrak{s}$ is the least cardinality of a splitting family on $\omega$. Naturally we are considering the following problem.

PROBLEM 2.2. Is the free algebra on $\mathfrak{s}$ generators a subalgebra of a representable algebra? Equivalently, is there a nowhere splitting independent family of cardinality $\mathfrak{s}$ ?

We show that both the hypotheses "there is an $\mathfrak{s}$-tower" and " $\mathfrak{b}=\mathfrak{s}$ " imply a "yes" answer to this problem.

THEOREM 2.3. If there is an $\mathfrak{s - t o w e r , ~ t h e n ~ t h e r e ~ i s ~ a ~ m a d ~ f a m i l y ~} \mathcal{M}$ such the free algebra on $\mathfrak{s}$ generators is a subalgebra of $\mathcal{B}_{\mathcal{M}}$.

P r o o f. By Lemma 1.1, let $\mathcal{I}$ be an $\mathfrak{s}$-tower such that $\mathcal{P}(\omega) / \mathcal{I}$ contains a copy of the free algebra on $\mathfrak{s}$ generators. Let $\left\{I_{\alpha}: \alpha<\mathfrak{s}\right\}$ be the generators for $\mathcal{I}$ which are increasing mod finite. Choose representatives, $\left\{X_{\alpha}: \alpha<\mathfrak{s}\right\}$, for the generators of the free algebra so that $X_{\alpha} \cap I_{\alpha}=\emptyset$ for each $\alpha<\mathfrak{s}$. Let $\mathcal{X}$ be the algebra generated by $\left\{X_{\alpha}: \alpha<\mathfrak{s}\right\}$.

Claim. The family $\mathcal{X}$ is nowhere splitting.

For each $\alpha<\mathfrak{s}$, let $\mathcal{X}_{\alpha} \subset \mathcal{P}\left(I_{\alpha}\right)$ be the algebra generated by $\left\{X_{\beta} \cap I_{\alpha}\right.$ : $\beta<\alpha\}$. Let $S$ be any infinite subset of $\omega$. Since $\mathcal{I}$ is a tower, there is an $\alpha<\mathfrak{s}$ such that $S \cap I_{\alpha}$ is infinite. By the definition of $\mathfrak{s}$, the family $\mathcal{X}_{\alpha}$ is nowhere splitting. Therefore, there is an infinite $Y \subset S \cap I_{\alpha}$ such that $Y$ is 
not split by any member of $\mathcal{X}_{\alpha}$. Finally, note that $\left\{X \cap I_{\alpha}: X \in \mathcal{X}\right\}=\mathcal{X}_{\alpha}$. This completes the proof of the claim and, by 2.1, the theorem.

THEOREM 2.4. If $\mathfrak{b}=\mathfrak{s}$, then there is a mad family $\mathcal{M}$ such that the free algebra on $\mathfrak{s}$ generators is a subalgebra of $\mathcal{B}_{\mathcal{M}}$.

Pr o of. Rather than working with $\mathcal{P}(\omega)$ it will be more convenient to use $\mathcal{P}(\omega \times \omega)$. Fix an unbounded family $\left\{f_{\alpha}: \alpha<\mathfrak{b}\right\}$ of increasing functions from $\omega$ to $\omega$. For $\alpha<\mathfrak{b}$, let $I_{\alpha}=\left\{(n, m): m<f_{\alpha}(n)\right\}$. Choose representatives, $\left\{X_{\alpha}: \alpha<\mathfrak{s}\right\} \subset \mathcal{P}(\omega)$, for the generators of the free algebra (as a subalgebra of $\mathcal{P}(\omega))$. For each $\alpha<\mathfrak{s}=\mathfrak{b}$, let

$$
Y_{\alpha}=\left[X_{\alpha} \times \omega\right] \backslash I_{\alpha} .
$$

Clearly, $\left\{Y_{\alpha}: \alpha<\mathfrak{s}\right\}$ is an independent family.

The algebra, $\mathcal{Y}$, generated by $\left\{Y_{\alpha}: \alpha<\mathfrak{s}\right\}$ is also nowhere splitting. Indeed, let $S \subset \omega \times \omega$. If there is an $n$ such that $S \cap(\{n\} \times \omega)$ is infinite, then, of course, this infinite subset of $S$ is not split by the family $\mathcal{Y}$ since $\{n\} \times \omega$ is not split by any member of $\mathcal{Y}$. If $\{n:(\{n\} \times \omega) \cap S \neq \emptyset\}$ is infinite, then, since $\left\{f_{\alpha}: \alpha<\mathfrak{s}\right\}$ is unbounded, we may choose $\alpha<\mathfrak{s}$ such that $S \cap I_{\alpha}$ is infinite. In this case we proceed exactly as in the previous theorem.

3. The topological setting. A subspace $Y$ of a topological space $X$ is said to be conditionally sequentially compact (abbreviated CSC) if every infinite subset of $Y$ has an infinite subsequence which converges to a point of $X$. Hagler asked (Top. Proc. 1978) for a compact Hausdorff space which has a countable dense CSC subspace but which is itself not sequentially compact (i.e. not CSC in itself). This was answered in the affirmative in [5] via a somewhat involved construction. It would be interesting to know if there is a cardinal $\kappa$ such that $2^{\kappa}$ serves as an answer to Hagler's question.

The cardinal number $\mathfrak{s}$ marks a watershed in this regard. It is the least cardinal $\kappa$ such that $2^{\kappa}$ is not sequentially compact ([3], [11]). So if some $2^{\kappa}$ serves as an answer to Hagler's question, $2^{\mathfrak{s}}$ does. As we will now show, this problem of whether $2^{\mathfrak{s}}$ has a countable dense CSC subspace is just Problem 2.2.

Proposition 3.1. If a compact space $X$ has a countable dense CSC subpace, then there is a $\Psi$-space $\Psi$ such that $\beta \Psi$ maps onto $X$.

Proof. Let $f$ be any bijection from $\omega$ to the dense CSC subspace of $X$. Let $\mathcal{M}$ be an almost disjoint family of subsets of $\omega$ with the property that for each $M \in \mathcal{M}, f(M)$ converges in $X$, and maximal with respect to this property. Since $f(\omega)$ is a CSC subspace of $X, \mathcal{M}$ is a mad family. Since $f$ extends to a continuous function from $\Psi(\mathcal{M})$ to $X$, the maximality of $\beta \Psi(\mathcal{M})$ guarantees that $f$ extends to all of $\beta \Psi(\mathcal{M})$. 
The above construction is flexible enough to show that many questions about compact Hausdorff space with a countable dense CSC subspace are most naturally answered by looking at $\Psi$-spaces.

Let us now add some more conditions to Proposition 2.1.

Proposition 3.2. Let $\kappa$ be an infinite cardinal. Each of the following are equivalent to each of the statements 2.1.1-2.1.3.

1. $2^{\kappa}$ has a countable dense CSC subspace.

2. There is a compact space $K$ with a countable dense CSC subspace such that $K$ maps onto $2^{\kappa}$.

3. There is a $\Psi$ such that $\beta \Psi$ maps onto $2^{\kappa}$.

P r o of. Dense CSC subspaces are clearly preserved by continuous surjections, so 3 implies 1 . Therefore the above three statements are equivalent by Proposition 3.1. It is easy to see that conditions 2.1.3 and 3.2.3 are equivalent.

Proposition 3.3. There is a model in which $\mathfrak{s}<\mathfrak{c}$, and yet $2^{\kappa}$ has a countable dense CSC subspace for each $\kappa \leq \mathfrak{c}$.

Proof. We may start with a model, $M$, of MA $+\neg \mathrm{CH}$ and use Theorem 1.4 to produce an independent family $\mathcal{X}=\left\{X_{\alpha}: \alpha<\mathfrak{c}\right\}$ such that there is a tower $\left\{T_{\alpha}: \alpha<\mathfrak{c}\right\}$ so that for each $\alpha<\mathfrak{c}, T_{\alpha} \cap X_{\alpha}=\emptyset$. We now add $\omega_{1}$ Cohen reals to obtain our model, $M[G]$. We claim that the family $\mathcal{X}$ remains nowhere splitting. Let us first recall the following two well-known results. First of all, any tower of $M$ remains a tower when Cohen reals are added. Secondly [8], if one Cohen real is added to $M$ then $M$ is again a model of $\mathfrak{p}=\mathfrak{c}$.

Now suppose that $Y \in M[G]$ is a subset of $\omega$. Since $\left\{T_{\alpha}: \alpha<\mathfrak{c}\right\}$ is a tower, we may choose an $\alpha<\mathfrak{c}$ such that $Y \cap T_{\alpha}$ is infinite. Next, we may view $Y$ as being obtained by the addition of just one Cohen real, hence we may apply $\mathfrak{p}=\mathfrak{c}$ in such an inner model to obtain an infinite $Z \subset Y \cap T_{\alpha}$ such that for every $\beta<\alpha$, either $Z \backslash X_{\beta}$ or $Z \cap X_{\beta}$ is finite. Since $Z \cap X_{\beta}$ is finite for all $\beta \geq \alpha$, it follows that $Z$ is not split by the family $\mathcal{X}$. If at least $\aleph_{1}$ Cohen reals are added, we have a model in which $\mathfrak{s}$ is $\omega_{1}$ [1].

Some other problems suggest themselves in the topological setting.

Problem 3.4. Is there a compact space, $K$, with a countable dense CSC subspace, and also an infinite closed subspace $Y$ such that $Y$ has no infinite converging sequences?

Problem 3.5. If $[0,1]^{\kappa}$ has a countable dense CSC subspace, must $2^{\kappa}$ also have one? 
In [5], it is shown that if there is a $K$ as in Problem 3.4, there is a $\Psi$ which has a compactification satisfying 3.4. However, we do not know if $\beta \Psi$ would be such a compactification.

Problem 3.6. If $\Psi$ has a compactification as in 3.4 , then is $\beta \Psi$ such a compactification?

Problem 3.7. Does $\mathfrak{s} \leq \mathfrak{b}$ guarantee that $2^{\mathfrak{s}}$ has a countable dense CSC subspace?

\section{References}

[1] B. Balcar, J. Pelant, and P. Simon, The space of ultrafilters on $N$ covered by nowhere dense sets, Fund. Math. 110 (1980), 11-24.

[2] J. E. Baumgartner and M. Weese, Partition algebras for almost-disjoint families, Trans. Amer. Math. Soc. 274 (1982), 619-630.

[3] D. Booth, A Boolean view of sequential compactness, Fund. Math. 85 (1974), 99102.

[4] A. Dow and R. Frankiewicz, Remarks on partitioner algebras, Proc. Amer. Math. Soc. 113 (1991), 1067-1070.

[5] A. Dow and P. Nyikos, Compact Hausdorff spaces with moderately large families of convergent sequences, preprint.

[6] S. Mr'owka, On completely regular spaces, Fund. Math. 41 (1954), 105-106.

[7] —, Some set-theoretic constructions in topology, ibid. 94 (1977), 83-92.

[8] J. Roitman, Adding a random or a Cohen real: topological consequences and the effect on Martin's axiom, ibid. 103 (1979), 47-60; Correction, ibid. 129 (1988), 141.

[9] F. Rothberger, On some problems of Hausdorff and of Sierpinski, ibid. 35 (1948), 29-46.

[10] J. Teresawa, Spaces $N \cup \mathcal{R}$ and their dimensions, Topology Appl. 11 (1980), 93-102.

[11] E. K. van Douwen, The integers and topology, in: Handbook of Set-Theoretic Topology, K. Kunen and J. Vaughan (eds.), North-Holland, 1984, 111-168.

[12] J. Vaughan, Small uncountable cardinals and topology, in: Open Problems in Topology, J. van Mill and G. M. Reed (eds.), North-Holland, 1990, 195-218.

DEPARTMENT OF MATHEMATICS

YORK UNIVERSITY

NORTH YORK, ONTARIO

CANADA M3J 1P3
DEPARTMENT OF MATHEMATICS UNIVERSITY OF SOUTH CAROLINA COLUMBIA, SOUTH CAROLINA 29208 U.S.A.

Received 21 January 1991;

in revised form 19 August 1991 